\title{
Preparing the Burns Unit to Accommodate Vaginal Delivery and Cesarean Section for Pregnant Women with COVID-19: A Successful Experience from Jordan
}

\author{
Omar Altal · Diab Bani Hani - Abdelwahab Aleshawi · \\ Ala"a Alhowary · Basil Obeidat · Majid Bani-Ata
}

Received: May 16, 2020 / Published online: August 27, 2020

(C) Springer Healthcare Ltd., part of Springer Nature 2020

Keywords: Burns unit; Cesarean section; COVID-19; Infection control; Women's health

\section{Key Summary Points}

COVID-19 is a highly infectious contagious virus.

One of the major concerns is crossinfection of healthcare staff and other patients without COVID-19 in the hospitals.

Digital Features To view digital features for this article go to https://doi.org/10.6084/m9.figshare.12690005.

\section{O. Altal $(\bowtie) \cdot$ B. Obeidat}

Department of Obstetrics and Gynecology, Faculty of Medicine, Jordan University of Science and Technology, Irbid 22110, Jordan

e-mail: altal_omar@yahoo.com

\section{Bani Hani · A. Alhowary}

Department of Anesthesia and Recovery, Faculty of Medicine, Jordan University of Science and Technology, Irbid 21110, Jordan

\section{A. Aleshawi}

Department of Special Surgery, Division of Ophthalmology, Faculty of Medicine, Jordan

University of Science and Technology, Irbid 21110 , Jordan

\section{Bani-Ata}

Department of Special Surgery, Division of Otolaryngology, Faculty of Medicine, Jordan University of Science and Technology, Irbid 21110, Jordan
This is a major problem for patients with COVID-19 who require a surgical or interventional procedure, especially pregnant women.

In our successful experience, we have adopted a "closed circuit" model for those patients by the proper utilization of the burns unit at our center. Accordingly, no cases of cross-infection were reported.

\section{INTRODUCTION}

The current coronavirus disease 2019 (COVID19) pandemic highlights the importance of a mindful utilization of financial and human resources. Preventing infections and preserving resources and manpower are crucial in healthcare. It is important to ensure the ability of obstetricians to function through the pandemic in dealing with pregnant women and their neonates. A tremendous effort should be made to minimize infection in this sector. In addition, more efforts should be conducted to monitor surgical patients, including pregnant women, with COVID-19 as they have a high mortality rate. A study by Lei et al. was conducted retrospectively on 34 patients with COVID-19 who underwent surgery. They reported that all patients developed COVID-19 
pneumonia shortly after surgery with abnormal findings on chest computed tomography scans, $15(44.1 \%)$ patients required admission to intensive care unit (ICU) during disease progression, and 7 patients $(20.5 \%)$ died after admission to ICU [1]. Chen et al. [2] reported that the risk of severe pneumonia in pregnant women can reach up $8 \%$, which is similar to that in the general population. However, there is currently no evidence for intrauterine infection caused by vertical transmission in women who develop COVID-19 pneumonia in late pregnancy. Pregnant women who developed COVID-19-associated pneumonia have an increased risk for obstetric complications (e.g., preterm labor, premature rupture of membranes, preeclampsia, cesarean section (CS)) $[3,4]$.

In addition, there is no agreed protocol regarding the appropriate place in dealing with pregnant women. A study was conducted in Italy about protocols for dealing with operative patients in the era of the COVID-19 pandemic. Coccolini et al. recommended that the designated COVID-19 operating areas must be allocated to the operating room closest to the entrance of the theater block entrance [5]. Also, they advised that patients' transit to and from the operating room must be as quick as possible and a pre-defined direct path must be kept as short as possible and away from other patients and people in general within the hospital in order to minimize the chances of infection. In addition, they recommended to reduce the number of operators and other staff as much as possible. At the end of the surgery, during the recovery phase, the patient must be assisted directly in the operation room until ready to be transferred back to the inpatients' place of stay. The time patients spend returning to wards must be reduced in order to minimize contact between patients with COVID-19 and the surrounding environment [5].

\section{PREPARING THE BURNS UNIT TO ACCOMMODATE THE DELIVERY}

The aforementioned protocols may carry a risk for contamination of hospital facilities and for cross-infection of healthcare staff and other admitted patients as the patients with COVID19 are transferred in an "open circuit". In addition, these protocols may affect the clinical status of these patients who, as reported by Lei et al. [1], are at increased risk of postoperative complications and death. Accordingly, at King Abdullah University Hospital in Jordan, we have prepared a "closed circuit" model for pregnant women and all surgical patients diagnosed with COVID-19. The model was discussed for all surgical patients by authors from our institution [6]. This "closed circuit" unit is the burns unit at our center. The burns unit is equipped with 10 rooms. Each room has a bed with negative pressure. Also, each room can be adjusted to become like ICU with intensive monitoring devices and mechanical ventilation. The rooms can be considered as a recovery room for each woman and a maternity unit. Moreover, the burns unit at our center has an operating theater that was supplied with the required obstetrical devices for vaginal delivery and for CS which include a neonatal coat, portable neonatal incubator, cardiotocography machine, endotracheal tubes, bulb suction, baby stopcock, masks, nasal cannula, cord clamp, umbilical vein catheter, the required medications, instrumental delivery devices, ultrasound, and all other neonate resuscitation devices. Furthermore, milk formulas and milk pump were preserved in the unit. Moreover, it is supplied with the required anesthetic equipment. All entrances were locked except for the main entrance which has a double electronic gate. The changing rooms and the shower bath are located between the double gate. Furthermore, the healthcare employees including nurses, midwives, neonatologists, anesthesiologists, and obstetricians are supplied with the necessary personal protection equipment including filtering face piece facial mask, disposable long-sleeve waterproof coats and gowns, two pairs of disposable nitrile gloves, protective goggles, disposable head caps, disposable long shoe covers, and alcohol hygiene. The healthcare employees reside in this unit in an arranged schedule. After the completion of the shifts, the healthcare employees are isolated for 14 days in special comfortable isolated 
Table 1 Clinical characteristics of two pregnant women with COVID-19 in Jordan

\begin{tabular}{|c|c|c|}
\hline Variable & Patient 1 & Patient 2 \\
\hline Age (years) & 28 & 30 \\
\hline Gestational week at admission & 37 & 36 \\
\hline Comorbidities & No & No \\
\hline Clinical presentation & Dry cough & Dry cough, headache, chills \\
\hline \multicolumn{3}{|l|}{ Vital sings at admission } \\
\hline Temperature $\left({ }^{\circ} \mathrm{C}\right)$ & 36.9 & 37.2 \\
\hline $\mathrm{SpO} 2(\%)$ & 95 & 96 \\
\hline Pulse rate & 91 & 110 \\
\hline Respiratory rate & 19 & 19 \\
\hline Blood pressure (mmHg) & $115 / 60$ & $112 / 70$ \\
\hline \multicolumn{3}{|l|}{ Laboratory investigations } \\
\hline WBC $(\times 1000 / \mathrm{mL})$ & 13 & 7.1 \\
\hline Hemoglobin $(\mathrm{g} / \mathrm{dL})$ & 11 & 9.3 \\
\hline Platelets $(\times 1000 / \mathrm{mL})$ & 365 & 100 \\
\hline $\mathrm{CRT}(\mu \mathrm{mol} / \mathrm{L})$ & 43 & 31 \\
\hline $\operatorname{ALT}(\mathrm{U} / \mathrm{L})$ & 7.3 & 5.4 \\
\hline $\operatorname{AST}(\mathrm{U} / \mathrm{L})$ & 15.6 & 11.6 \\
\hline Albumin $(\mathrm{g} / \mathrm{L})$ & 39 & 35 \\
\hline $\mathrm{CRP}(\mathrm{mg} / \mathrm{L})$ & 7.1 & 10 \\
\hline $\operatorname{ESR}(\mathrm{mm} / \mathrm{h})$ & 48 & 35 \\
\hline Antepartum complications & No & No \\
\hline Delivery & Delivered by CS & Delivered by CS \\
\hline Gestational age at delivery & 39 & 38 \\
\hline APGAR score & 9 & 9 \\
\hline Neonatal SARS-CoV-2 status & Negative & Negative \\
\hline
\end{tabular}

$C R P$ C-reactive protein, ESR erythrocyte sedimentation rate, $W B C$ white blood count, $C R T$ creatinine, $A L T$ alanine aminotransferase, $A S T$ aspartate aminotransferase

facilities without contacting anybody and they are offered COVID-19 tests before the end of the isolation. The center has an intermediate care unit which was prepared for any burns case.

\section{MANAGEMENT OF TWO PREGNANT WOMEN}

Two CS under spinal anesthesia were successfully performed for two pregnant women. The 


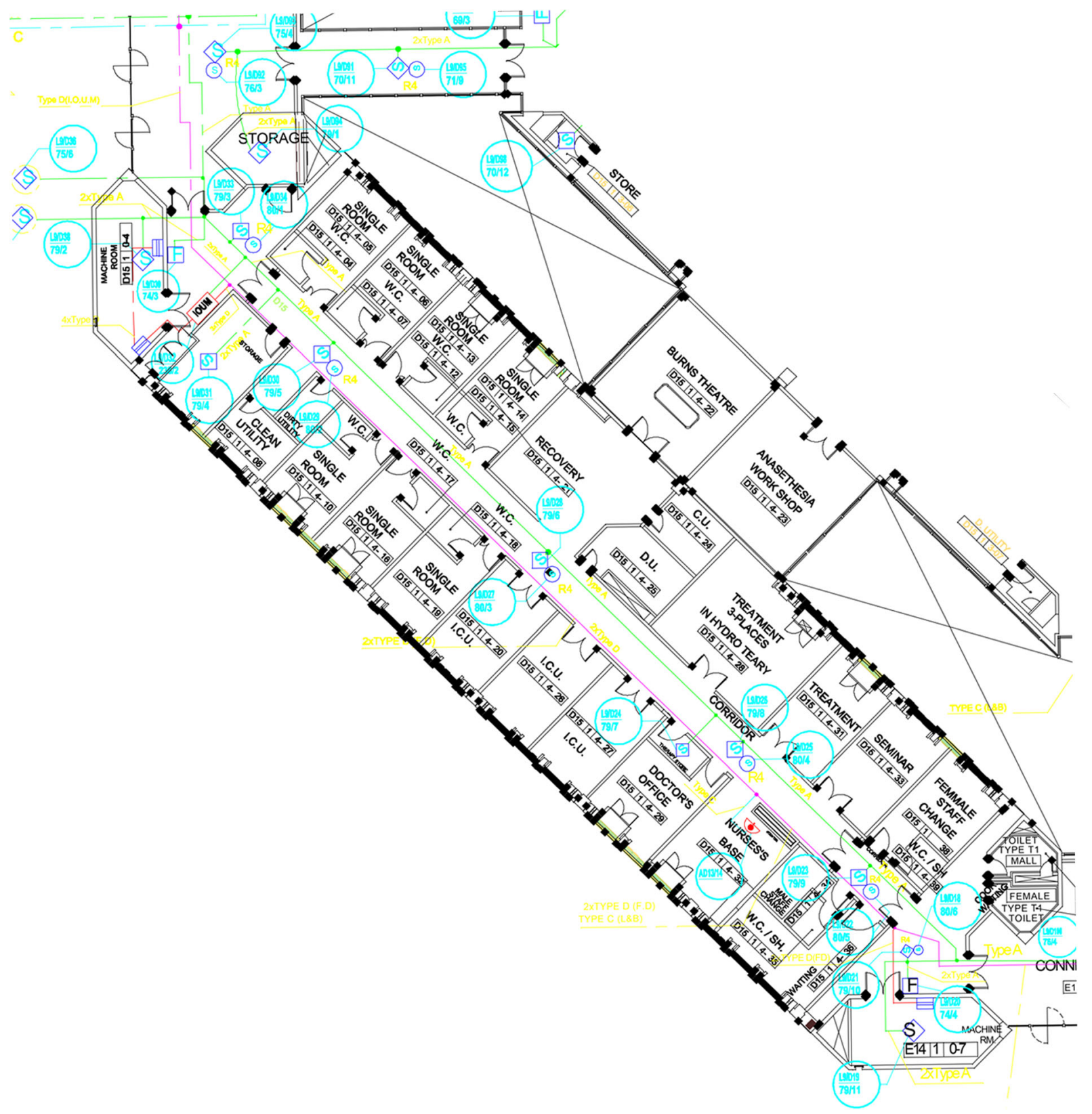

Fig. 1 Blueprint for the burns unit indicating the presence of 10 isolated rooms and operation room

women's gestational ages at admission were 36 and 37 weeks. The clinical characteristics for both women are summarized in Table 1. It was suggested that both women wait for 2 weeks before proceeding with delivery in the hope of achieving recovery from the virus; however, after around 2 weeks after admission, COVD-19 tests were still positive for both women. Accordingly, two CS were performed at the gestational age of 38 and 39 weeks. The two CS were conducted under spinal anesthesia and took approximately $40 \mathrm{~min}$. Eight healthcare employees participated in the operations including a consultant obstetrician, obstetrics resident, consultant anesthesiologist with his assistant, two operation nurses, neonatologist, and midwife. The complete anesthetic management for one of the two cases was described 
in detail by one of the authors [7]. The neonates were admitted in other prepared rooms in the burns unit. These rooms have special portable neonatal incubators that serve as neonatal intensive care beds. Also, the rooms were supplied with the required milk formula which was administered by the midwife who resided in the burns unit. The neonatologist was on-call ready for any urgent issue. In addition to amniotic analysis, the neonates underwent swab collection for COVID-19. These tests were repeated three times and the results were normal for both neonates. The neonates resided in the burns unit in their special rooms with the care of the midwife until the mothers recovered from the virus (about 1-2 weeks after the operation). It is important to highlight that the instruments from this unit have their own releasing track controlled by the infection control team. We did not find any cross-infection of any of the healthcare staff or any other admitted seronegative patient.

Figure 1 demonstrates the blueprint of the burns unit at our center.

This study was approved by King Abdullah University Hospital/Jordan University of Science and Technology. All patients provided informed consent to participate and the study was conducted in accordance with the Declaration of Helsinki.

Cross-infection among healthcare staff and hospital facilities is a major concern which can affect any healthcare systems in the world. Proper management for the resources is recommended in order to empower the healthcare staff against the COVID-19 pandemic.

\section{ACKNOWLEDGEMENTS}

We would like to thank the midwife Ms. Rasha Alkhattib for her efforts

Funding. No funding or sponsorship was received for this study or publication of this article.

Authorship. All named authors meet the International Committee of Medical Journal
Editors (ICMJE) criteria for authorship for this article, take responsibility for the integrity of the work as a whole, and have given their approval for this version to be published.

Disclosures. Omar Altal, Diab Bani Hani, Abdelwahab Aleshawi, Ala"a Alhowary, Basil Obeidat and Majid Bani-Ata have nothing to disclose.

Compliance with Ethics Guidelines. This study was approved by King Abdullah University Hospital/Jordan University of Science and Technology. All patients provided informed consent to participate and study was conducted in accordance with the Declaration of Helsinki.

\section{REFERENCES}

1. Lei S, Jiang F, Su W, et al. Clinical characteristics and outcomes of patients undergoing surgeries during the incubation period of COVID-19 infection. EClinicalMedicine. 2020;2020:100331. https://doi.org/10. 1016/j.eclinm.2020.100331.

2. Chen L, Li Q, Zheng D, et al. Clinical characteristics of pregnant women with Covid-19 in Wuhan, China. N Engl J Med. 2020. https://doi.org/10.1056/ NEJMc2009226.

3. Rothe C, Schunk M, Sothmann P, et al. Transmission of 2019-nCoV infection from an asymptomatic contact in Germany. N Engl J Med. 2020;3(82):970-1.

4. Schwartz DA. An analysis of 38 pregnant women with COVID-19, their newborn infants, and maternal-fetal transmission of SARS-CoV-2: maternal coronavirus infections and pregnancy outcomes. Arch Pathol Lab Med. 2020;144(7):799-805.

5. Coccolini F, Perrone G, Chiarugi M, et al. Surgery in COVID-19 patients: operational directives. World J Emerg Surg. 2020;15(1):25. https://doi.org/10.1186/ s13017-020-00307-2.

6. Bani-Hani D, Altal O, Aleshawi A, et al. Expanding access for COVID-19 patients by transforming a burn unit into a closed-circuit unit for surgical patients: experience from an academic medical center in Jordan. Patient Saf Surg. 2020;14:25.

7. Bani Hani D, Alsharaydeh I, Bataineh A, et al. Successful anesthetic management in Cesarean section for pregnant woman with COVID-19. Am J Case Rep. 2020;21:e925512. 